\title{
NMR Study of Vacancy and Structure-Induced Changes in $\mathrm{Cu}_{2-x} \mathrm{Te}$
}

\author{
Ali A. Sirusi ${ }^{\mathrm{a}}$, Alexander Page ${ }^{\mathrm{b}}$, Ctirad Uher $^{\mathrm{b}}$, Joseph H. Ross, Jr. ${ }^{\mathrm{a}, \mathrm{c}, *}$ \\ ${ }^{a}$ Department of Physics and Astronomy, Texas A $6 M$ University, College Station, Texas 77843, USA \\ ${ }^{b}$ Department of Physics, University of Michigan, Ann Arbor, MI 48109, USA \\ ${ }^{c}$ Department of Materials Science and Engineering, Texas A\&M University, College Station, Texas 77843, USA
}

\begin{abstract}
We report $\mathrm{Cu}$ and Te NMR measurements on $\mathrm{Cu}_{2-x}$ Te with $x$ between 0.13 and 0.22 . Aided by powder x-ray analysis and computed NMR quadrupole shifts, a structure change near $x=0.20$ was found consistent with structures reported by Baranova, with best fits to the $\beta$-I structure for $x=0.22$ and $\beta$-III for smaller $x$. NMR $T_{1}$ and Hall effect results demonstrate $p$-type electronic behavior with filling of simple hole pockets induced by increased numbers of vacancies for both phases. Furthermore the $\mathrm{Cu}$ and Te chemical shifts are large and negative, as observed in topologically inverted semiconductors, with a splitting into two distinct local environments for both $\mathrm{Cu}$ and Te sites in the $x=0.22$ structure. $\mathrm{Cu} T_{1}$ results show a rapid decrease of the energy barrier for initiation of $\mathrm{Cu}$ ion hopping to $0.12 \mathrm{eV}$ for $x=0.22$, considerably smaller than observed at higher temperatures, indicating a tail of relatively mobile $\mathrm{Cu}$ ions which may be of significance for potential device applications.
\end{abstract}

Keywords: Nuclear Magnetic Resonance, thermoelectric materials, superionic conductor, topological electronic behavior

\section{Introduction}

$\mathrm{Cu}_{2-x} \mathrm{Te}$ has been of interest as a promising candidate in thermoelectric [1-4] and solar cell $[5,6]$ applications, and even for cancer treatment [7].

5 Moreover, it was recently shown to have characteristics associated with topologically nontrivial band inversion [8] and that if formed as a 2D crystal it may exhibit topological insulator characteristics [9]. Topological insulator behavior is also well es10 tablished [10-13] in $\mathrm{Ag}_{2} \mathrm{Se}$ and $\mathrm{Ag}_{2} \mathrm{Te}$. However the $\mathrm{Cu}_{2-x}$ Te phase diagram includes structures not fully determined in all cases, and there are several phase transitions at high temperatures $[14,15]$ as the Te lattice adapts to superionic motion of the $\mathrm{Cu}$ ions [16]. The ionic mobility can also induce detrimental effects for device applications. Thus it is important to better understand the structures and their relation to the lattice dynamics and electronic behavior.

$\mathrm{Cu}_{2-x} \mathrm{Te}$ tends to form spontaneously having nonzero $x$, and the reported structures are depen-

\footnotetext{
*Corresponding author. Tel: +1-979-845-7823

Email address: alisirusi@tamu.edu (Ali A. Sirusi)
}

dent on the $\mathrm{Cu}$ vacancy density. Initially, Nowotny [17] reported a hexagonal structure in space group $P 6 / \mathrm{mmm}$ with lattice parameters $a_{0}=4.237 \AA$, ${ }_{25} c_{0}=7.274 \AA$, and $Z=2$. This structure consists of interconnected $\mathrm{Cu}$-Te layers arranged as 2dimensional sheets, as shown as an inset in Fig. 1. Later, stoichiometric $\mathrm{Cu}_{2} \mathrm{Te}$ was indicated to have a larger superstructure based on the hexagonal struc30 ture [18] or an orthorhombic phase [15]. It has also been reported that the structure is trigonal $[19,20]$ with space group $P \overline{3} m 1$, and a recent report [21] predicted that the $P \overline{3} m 1$ trigonal phase is stable extending well into the range $x \neq 0$. The phase region $0 \leq x \leq 0.1$ was also described to consist at room temperature of two phases: hexagonal and orthorhombic [14], while recently it was predicted from simulations that $\mathrm{Cu}_{2} \mathrm{Te}$ crystallizes in a monoclinic [6] structure with 4 formula units per cell.

40 Baranova [22-25] reported several specific structures vs. vacancy concentration: $\beta$-I (trigonal with space group $P 3 m 1$, a fully-occupied phase with stoichiometry $\mathrm{Cu}_{7} \mathrm{Te}_{4}=\mathrm{Cu}_{1.75} \mathrm{Te}$ ), $\beta$-II (hexagonal, space group $P \overline{6} m 2$, having partial occupancy also 45 giving composition $\mathrm{Cu}_{1.75} \mathrm{Te}$ ), and $\beta$-III (trigonal, 


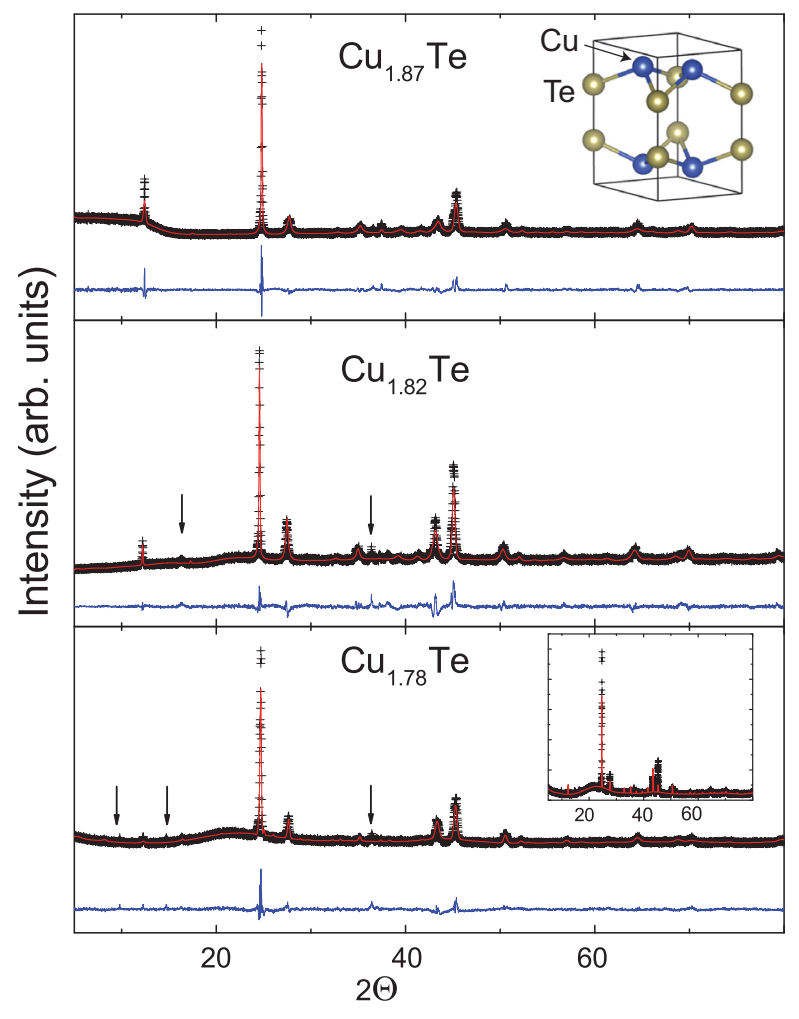

Figure 1: XRD patterns of $\mathrm{Cu}_{1.87} \mathrm{Te}, \mathrm{Cu}_{1.82} \mathrm{Te}$, and $\mathrm{Cu}_{1.78} \mathrm{Te}$ samples, with fittings to $P \overline{3} m 1$ trigonal structure (first two samples) and $\beta$-II structure (for $\mathrm{Cu}_{1.78} \mathrm{Te}$ ). Lower inset: $\mathrm{Cu}_{1.78}$ Te with fitting to $\beta$-I structure. Red lines are fitted curves and blue lines show the differences. Arrows are extra peaks noted in the text. Upper inset: Nowotny structure; fitted structures are larger superstructures with similar layered structure. space group $P 3 m 1$, partial occupancy corresponding to $\mathrm{Cu}_{1.812} \mathrm{Te}$ ). These structures have 6,7 , and 24 distinct $\mathrm{Cu}$ sites and 4, 3 and 12 Te sites per cell, for $\beta$-I, $\beta$-II, and $\beta$-III respectively, while the previ50 ously mentioned $P \overline{3} m 1$ trigonal phase has $4 \mathrm{Cu}$ and 2 Te sites/cell. These structures have closely related lattice parameters, and may be considered to be superstructures of Nowotny's hexagonal structure, with all containing interconnected $\mathrm{Cu}$-Te atomic

55 layers, although the nearest neighbors within the layers and site symmetries differ considerably.

In this report we describe $\mathrm{Cu}$ and Te NMR measurements on three $\mathrm{Cu}_{2-x}$ Te samples of varying composition from $x=0.13$ to 0.22 , supported by

${ }_{60}$ Hall coefficient and structural characterization, and all-electron computation. The samples are in the composition range of this material previously identified as having topologically nontrivial band inversion [8]. A structure change in the range $x=0.20$

65 induces a large change in NMR spectra, and we find that the charge carriers can be accounted for in a relatively straightforward way, as a heavily doped semiconductor with holes contributed by $\mathrm{Cu}$ vacancies. Furthermore the barriers for low-temperature $70 \mathrm{Cu}$ hopping become progressively smaller as the vacancy density increases, with a very small barrier obtained for $x=0.22$.

\section{Experimental and Computational meth- ods}

\subsection{Synthesis and Experimental Methods}

Sample ingots were made by melting followed by annealing. First, Cu shot (Alfa Aesar 99.9999\%) and Te pieces (Alfa Aesar 99.9999\%) totaling 15 grams were sealed in vacuum in a graphite coated 30 quartz tube, with a mole ratio corresponding to $\mathrm{Cu}_{2} \mathrm{Te}$. The tube was heated over a period of seven hours to $1393 \mathrm{~K}$, then held there for three hours and naturally cooled to room temperature. The resulting ingot was polished and then powderized in an agate mortar and pestle. The powder was divided into three parts and cold pressed into pellets, which were placed in separate evacuated quartz tubes and annealed at $750 \mathrm{~K}, 850 \mathrm{~K}$, and $1000 \mathrm{~K}$ for one week. In each case, a heating rate of $50 \mathrm{C} / \mathrm{hr}$ and a cool-

90 ing rate of $25 \mathrm{C} / \mathrm{hr}$ was used for annealing. The ingots from this process were then polished and cut into bars for further measurements.

In backscattered electron images collected using a Cameca SXFive Electron Probe Micro-Analyzer, 

ondary phases detected. This was followed by wavelength dispersion spectroscopy (WDS) measurements on at least four points on each sample, with compositions from each point showing variations of 0.3 at.\% or less. From the mean values, the WDS results showed the samples annealed at 750 , 850 , and $1000 \mathrm{~K}$ to have compositions $\mathrm{Cu}_{1.78} \mathrm{Te}$, $\mathrm{Cu}_{1.82} \mathrm{Te}$, and $\mathrm{Cu}_{1.87} \mathrm{Te}$ respectively. The samples are denoted below according to these compositions.

As noted above, $\mathrm{Cu}_{2-x}$ Te tends to form spontaneously with a $\mathrm{Cu}$ deficit $(x>0)$, and apparently this occurs during the initial formation of the ingot from the melt. The different compositions after the anneal processes can be understood to occur due to preferential evaporation of Te due to its larger vapor pressure.

Structural characterization via powder $\mathrm{x}$-ray diffraction (XRD) was performed on a Bruker D8 $\mathrm{X}$-ray Powder Diffractometer with $\mathrm{Cu} \mathrm{K} \alpha$ radiation. To analyze the results Rietveld refinements were performed using EXPGUI software [26, 27]. The Hall coefficient was measured at ambient temperature using an $\mathrm{AC}$ 4-probe method in a custom built apparatus under a maximum field of 1

${ }_{120}$ Tesla, and also at low temperatures using a Quantum Design, Inc. MPMS combined with a homebuilt bridge

The NMR techniques were similar to those described elsewhere [8], with echo integration used to in static line shapes in a fixed 9 T field. Solid $\mathrm{CuCl}$ and aqueous $\mathrm{Te}(\mathrm{OH})_{6}$ standards were used for $\mathrm{Cu}$ and $\mathrm{Te}$ references with positive relative shifts 175 having paramagnetic sign ( $\delta$ convention). $\mathrm{Te}(\mathrm{OH})_{6}$ has a shift of $+707 \mathrm{ppm}$ relative to the primary 130 standard [28], $\left(\mathrm{CH}_{3}\right)_{2} \mathrm{Te}$, and the spectra have been adjusted accordingly. ${ }^{63} \mathrm{Cu}$ NMR spectra were fitted using the DmFit package [29]. The chemical 180 shift $\left(\delta_{c s}\right)$ and Knight shift $(K)$ were defined relative to the total shift $(\delta)$ according to $\delta=\delta_{c s}+K$, 135 with $K$ identified according to metallic shift theory, as representing the electron spin contribution in absence of spin-orbit coupling.

NMR spin-lattice relaxation $\left(T_{1}\right)$ measurements used an inversion-recovery sequence, with $T_{1}$ as fitting parameter within a multi-exponential magnetic relaxation function $\left[M\left(t_{\text {wait }}\right) / M(0)=1-\right.$ $\left.\alpha\left(0.1 e^{-t_{\text {wait }} / T_{1}}+0.9 e^{-6 t_{\text {wait }} / T_{1}}\right)\right]$, as previously re- 190 ported $[8]$ for the ${ }^{63} \mathrm{Cu}$ and ${ }^{65} \mathrm{Cu}$ central transitions. At $77 \mathrm{~K}$ a small quadrupole contribution to $1 / T_{1}$ the nuclear magnetic and quadrupole moments. For ${ }^{125} \mathrm{Te}$, which has spin $1 / 2$, we used the function $\left[M\left(t_{\text {wait }}\right) / M(0)=1-\alpha e^{-t_{\text {wait }} / T_{1}}\right]$.

\subsection{Computational Methods}

Density functional theory (DFT) calculations were carried out using the WIEN2k code $[31,32]$ to determine electric field gradients (EFGs) and model the $\mathrm{Cu}$ NMR spectra, and for energy minimization of the structures. These were performed with the Generalized Gradient Approximation (GGA) using the PBE exchange potential [33], with 200 $k$-points, and $\mathrm{Rk}_{\max }=7$. Calculations done on reported $\mathrm{Cu}_{2} \mathrm{Te}$ structures used experimental lattice constants from the literature, but with internal parameters minimized. Although in some cases partial occupation of $\mathrm{Cu}$ sites was given in reported structures, for DFT computations we set occupation numbers equal to 1 (compositions $\mathrm{Cu}_{2} \mathrm{Te}$ except for $\beta$-I, which is $\mathrm{Cu}_{7} \mathrm{Te}_{4}$ with all sites filled).

\section{Results and Analysis}

\subsection{Structure and Transport Results}

XRD patterns are shown in Fig. 1. A prominent feature is that with lower annealing temperature and increased vacancy density the peak intensity at $2 \Theta=12.3^{\circ}$ is reduced. This is accompanied by appearance of several small additional peaks as shown in the figure (arrows). The XRD patterns for $\mathrm{Cu}_{1.87} \mathrm{Te}$ and $\mathrm{Cu}_{1.82} \mathrm{Te}$ have similar features and were fitted using the $P \overline{3} m 1$ trigonal structure [19-21], while the $\mathrm{Cu}_{1.78}$ Te result was fitted using the $\beta$-II hexagonal structure [24]. For $\mathrm{Cu}_{1.87} \mathrm{Te}$ and $\mathrm{Cu}_{1.82} \mathrm{Te}$, internal structural parameters were taken from Ref. [21] with site occupations set to $100 \%$, since the powder fits were found not very sensitive to the partial occupancy of $\mathrm{Cu}$ sites as reported. Refinements of the unit cell (trigonal space group $P \overline{3} m 1, \# 164)$ yielded lattice parameters $a=8.362$ and $c=7.219 \AA$ for $\mathrm{Cu}_{1.87} \mathrm{Te}$, and $a=8.365$ and $c=7.226 \AA$ for $\mathrm{Cu}_{1.82} \mathrm{Te}$, similar to the reported [19-21] $a=8.37$ and $c=7.16 \AA$ for this structure. Fitted cell volumes are 109.3 and $109.5 \AA^{3}$, respectively when reduced by $1 / 4$ corresponding to the underlying $Z=2$ Nowotny hexagonal cell. However note that the $\beta$-III structure of Baranova worked equally well (not shown) in fitting the XRD patterns for these two samples, with suitable adjustment of the reported [22] lattice parameters $a=8.37$ and $c=21.60 \AA$, representing in 
this case a larger superstructure with the trigonal space group P3m1 (\#156). Thus as has been noted previously with powder XRD alone it is difficult to distinguish these structures.

$\mathrm{Cu}_{1.78}$ Te refinement yielded $a=4.161$ and $c=$ $21.60 \AA$ in the $P \overline{6} m 2$ (\#187) $\beta$-II structure, also with all $\mathrm{Cu}$ sites set to $100 \%$ filled. This is comparable to the reported [24] $a=4.17$ and $c=21.65$ $\AA$. The refined values yield a reduced cell volume of $107.9 \AA^{3}$ when multiplied by $1 / 3$ based on the $c$-direction supercell. Compared to the other two samples the lattice parameters and cell volume are thus significantly smaller, reflecting the change in structure going to $x=0.22$. Changes in NMR spectra described below further demonstrate the differing structures for these compositions, a result at variance with the computation-based result [21] that the $P \overline{3} m 1$ trigonal structure has the lower energy for the entire composition range. We found that the alternative $\beta$-I, $\mathrm{Cu}_{7} \mathrm{Te}_{4}$ structure proposed for this region of the phase diagram [23] could also be indexed to the XRD pattern, but with some intensity differences involving the reflections near 41 and $42^{\circ}$ (inset, Fig. 1).

Hall measurements indicated all $\mathrm{Cu}_{2-x}$ Te samples to be heavily-doped $p$-type, with hole concentrations scaling with $\mathrm{Cu}$ vacancy concentration $x$. Results at ambient temperature correspond to hole concentrations $p=6.5 \times 10^{21}, 4.1 \times 10^{21}$, and 3.6 $\times 10^{21} \mathrm{~cm}^{-3}$ for $\mathrm{Cu}_{1.78} \mathrm{Te}, \mathrm{Cu}_{1.82} \mathrm{Te}$, and $\mathrm{Cu}_{1.87} \mathrm{Te}$, For $\mathrm{Cu}_{1.82} \mathrm{Te}$ and $\mathrm{Cu}_{1.87} \mathrm{Te}$ measurements were also performed down to liquid helium temperatures, with results showing a variation in $p 255$ of about $10 \%$ vs. temperature, with a weak plateau near $100 \mathrm{~K}$ in a manner very similar to what was 230 reported previously in this phase range [3].

Estimated carrier concentrations, assuming one hole per $\mathrm{Cu}$ vacancy, can be obtained using the fit- 260 ted XRD cell volumes. This leads to $p=4.1 \times 10^{21}$, $3.3 \times 10^{21}$, and $2.4 \times 10^{21} \mathrm{~cm}^{-3}$ for the $\mathrm{Cu}_{1.78} \mathrm{Te}$,

${ }_{235} \mathrm{Cu}_{1.82} \mathrm{Te}$, and $\mathrm{Cu}_{1.87} \mathrm{Te}$ samples, respectively. The Hall results thus scale with composition, pointing to the filling of hole pockets starting with the parent 265 composition $\mathrm{Cu}_{2} \mathrm{Te}$ as a semiconductor. There has been uncertainty about this, with calculated electronic properties generally reporting to give metallic or semimetallic behavior [34, 35]. The latter may be due to density functional methods which underestimate the gap [8] and/or uncertainty in the correct structure. The results also point to carriers numbering more than one per vacancy, which may be related to partial covalency of $\mathrm{Cu}$-Te bonding, a

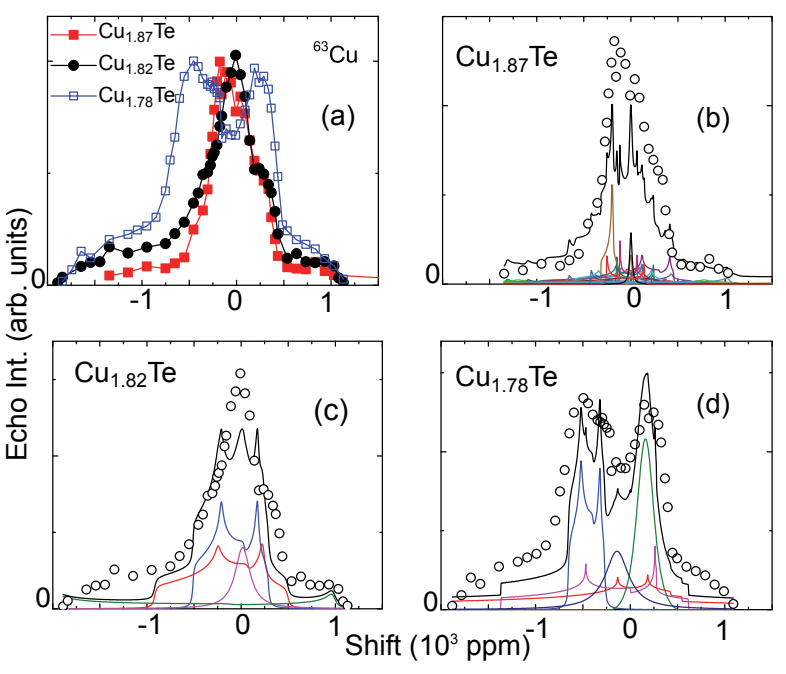

Figure 2: (a) ${ }^{63} \mathrm{Cu}$ spectra of different samples measured at $290 \mathrm{~K}$. (b)-(d): individual spectra along with DFTcalculated line shapes described in the text, for: (b) $\beta$-III structure of Baranova [22], (c) $P \overline{3} m 1$ trigonal structure, and (d) $P 3 m 1 \beta$-I structure [24].

result also previously indicated by calculations for the $\mathrm{Cu}_{2} \mathrm{Te}$ Nowotny structure [34]. Removing $\mathrm{Cu}$ can in this case leave dangling bonds which may remove more than one electron from the valence band.

\section{2. $C u$ NMR Results}

${ }^{63} \mathrm{Cu} \mathrm{NMR}$ spectra obtained at $290 \mathrm{~K}$ are shown in Fig. 2(a). Each spectrum consists of superposed quadrupole-broadened central transitions $(1 / 2$ to $-1 / 2$ ) for each site. The $\mathrm{Cu}_{1.87} \mathrm{Te}$ and $\mathrm{Cu}_{1.82} \mathrm{Te}$ results are similar to those reported recently for a similar composition [8], however the $\mathrm{Cu}_{1.78}$ Te spectrum exhibits a distinctive splitting with two main peaks, corresponding to its different structure as outlined above.

${ }^{63} \mathrm{Cu}$ spin-lattice relaxation times $\left(T_{1}\right)$ vs. temperature are shown in Fig. 3, measured at the peak amplitude positions for $\mathrm{Cu}_{1.87} \mathrm{Te}$ and $\mathrm{Cu}_{1.82} \mathrm{Te}$, and at a position between the two prominent peaks for $\mathrm{Cu}_{1.78} \mathrm{Te} .{ }^{65} \mathrm{Cu} T_{1}$ measurements were also performed at fixed temperatures of $4.2 \mathrm{~K}, 77 \mathrm{~K}$, and $290 \mathrm{~K}$, allowing the relative contributions due to electric quadrupole vs. magnetic mechanisms to be assessed. For all three samples it was found that a magnetic mechanism strongly dominates at $77 \mathrm{~K}$, with the ratios ${ }^{63} T_{1} /{ }^{65} T_{1}$ equal to 1.13 , 1.12 , and 1.14 respectively for $\mathrm{Cu}_{1.87} \mathrm{Te}, \mathrm{Cu}_{1.82} \mathrm{Te}$, and $\mathrm{Cu}_{1.78} \mathrm{Te}$, compared the the expected ratio [8] 


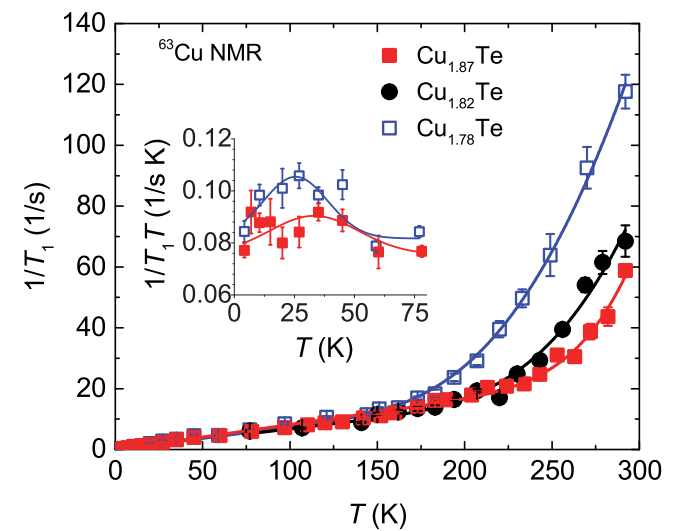

Figure 3: ${ }^{63} \mathrm{Cu} \mathrm{1/T}$ vs temperature for the three samples as indicated. Solid curves are fits as explained in the text. Inset: $1 / T_{1} T$ vs. $T$ for $\mathrm{Cu}_{1.87} \mathrm{Te}$ and $\mathrm{Cu}_{1.78} \mathrm{Te}$ at low temperatures with curves: guides to the eye.

${ }^{65} \gamma^{2} /{ }^{63} \gamma^{2}=1.14$, vs. ${ }^{65} Q^{2} /{ }^{63} Q^{2}=0.85$ for a pure quadrupole relaxation mechanism. Thus we analyzed the $77 \mathrm{~K}$ results according to a Korringa mechanism due to metallic carriers [36]. On the other hand the ratios indicate that a quadrupole mechanism becomes dominant at $290 \mathrm{~K}$ as ionic hopping becomes enhanced, in good agreement with previously reported results [8]. Futhermore there is a small departure from $1 / T_{1} T=$ constant behavior below about $50 \mathrm{~K}$ for $\mathrm{Cu}_{1.78} \mathrm{Te}$ (inset, Fig. 3), and the ${ }^{63} \mathrm{Cu} /{ }^{65} \mathrm{Cu}$ ratios at $4 \mathrm{~K}$ also indicate reappearance of a quadrupole contribution, signaling perhaps an effect of local vibrational modes with strong anharmonicity [37]. As shown in the inset, the effect becomes small or absent for the $\mathrm{Cu}_{1.87} \mathrm{Te}$ sample. There has been previous interest in lowenergy modes exhibited by other superionic conductors [38].

Analyzing for ionic motion approaching room 345 temperature, $1 / T_{1}$ could be fitted over the full range to $f \exp \left(-\Delta E / k_{\mathrm{B}} T\right)+B T$, where $\Delta E$ is the activation energy and $B T$ represents primarily the metallic contribution. Least squares fitting (solid curves in Fig. 3) gave $\Delta E=0.21,0.16$, and $0.12 \mathrm{eV} 350$ for $\mathrm{Cu}_{1.87} \mathrm{Te}, \mathrm{Cu}_{1.82} \mathrm{Te}$, and $\mathrm{Cu}_{1.78} \mathrm{Te}$, respectively, with $\Delta E$ thus becoming strongly reduced with increasing vacancy density, and with the change in superstructure going to $\mathrm{Cu}_{1.78} \mathrm{Te}$. These activation energies are smaller than have been reported from 355 higher-temperature measurements [39, 40], indicat305 ing a population of ions that is particularly mo- bile at low $T$. Also the results are consistently smaller than found in the previous NMR measurement [8], the latter involving a sample formed by spark plasma sintering, indicating perhaps that refinement by spark plasma sintering helps to immobilize $\mathrm{Cu}$ ions. The $\mathrm{Cu}_{1.78}$ Te sample with lowest barrier also exhibited the peak in low temperature $1 / T_{1} T$, and thus the small $\mathrm{Cu}$-ion hopping barriers in this sample may also be connected to the presence of low energy localized vibrational modes.

Regarding the magnetic contribution $\left(T_{1 M}\right)$ dominant at $77 \mathrm{~K}$, note that for the measured carrier densities, assuming parabolic hole pockets, as previously shown [8] the doping is well into the metallic 320 regime. Thus Korringa behavior $\left(K^{2} T_{1 M} T=\right.$ constant) will apply, with $K=$ Knight shift. For the simple metallic case, or as long as correlation effects remain independent of $p[36,41], 1 / T_{1 M} T \propto$ $g^{2}\left(E_{F}\right)$, and $g\left(E_{F}\right) \propto \sqrt{E_{F}} \propto p^{1 / 3}$, where $g\left(E_{F}\right)$ is the Fermi-level electronic density of states and $p$ is the hole density. Therefore $1 / T_{1 M}$ vs. $p^{2 / 3}$ should be linearly related. Fig. 4 shows such a plot, with $1 / T_{1 Q}$ contributions ( $10 \%$ or less relative to $\left.1 / T_{1 M}\right)$ subtracted as described above. Carrier densities plotted are the measured values for $77 \mathrm{~K}$, except for $\mathrm{Cu}_{1.78} \mathrm{Te}$. The previously reported value [8] has been included in the plot as an open circle, and it is seen that the results fall in line as expected, including the $\mathrm{Cu}_{1.78} \mathrm{Te}$ result for which the superstructure has changed. Together with the Hall results this indicates carriers filling a single parabolic hole pocket with no compensation. As noted above, DFT calculations [42] in the Nowotny structure have indicated the material to be metallic with multiple band crossings, but these results are likely affected by DFT methods underestimating the gap and/or uncertainty in crystal structure.

Fitting the central regions of the $\mathrm{Cu}_{1.87} \mathrm{Te}$ and $\mathrm{Cu}_{1.82} \mathrm{Te}{ }^{63} \mathrm{Cu}$ spectra with Gaussian curves yields an approximate measure of their shift positions, $-50 \mathrm{ppm}$ and $-24 \mathrm{ppm}$ respectively, making an assumption as before [8] that the sharp peak is contributed mainly by a site with small spectral width. Using measured $1 / T_{1}$ values as described above, and assuming $K^{2} T_{1} T=3.7 \times 10^{-6} \mathrm{sK}$, for ${ }^{63} \mathrm{Cu}$ in the simple metallic limit, we obtain $K=520$ and $490 \mathrm{ppm}$, and hence $\delta_{c s}=-570$ and $-510 \mathrm{ppm}$, respectively, close to the estimated $\delta_{c s}=-490$ ppm peak position of the previous sample [8]. A similar analysis for $\mathrm{Cu}_{1.78}$ Te yields $K=600$ and $570 \mathrm{ppm}$ for the two peaks, an indication that the much larger splitting observed for this composition 


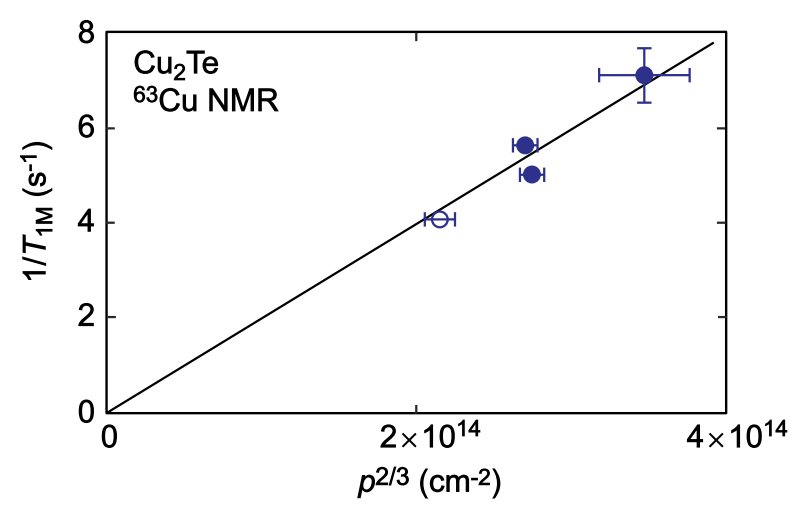

Figure $4:{ }^{63} \mathrm{Cu} 1 / T_{1 M}$ for $T=77 \mathrm{~K}$, plotted vs $p^{2 / 3}$, where $p$ is the measured hole density. Open circle: previously reported value [8]. Straight line is least-squares fitted line through zero. Vertical error bars for $\mathrm{Cu}_{1.78}$ Te sample encompasses range of measured values, including measurements at two peaks in spectrum and at line shape center.

is due instead to differences in chemical shift and/or quadrupole contributions.

\subsection{Te NMR Results}

Fig. 5 shows ${ }^{125}$ Te spectra at $77 \mathrm{~K}$. The line shape and overall shift for $\mathrm{Cu}_{1.87} \mathrm{Te}$ is essentially identical to the previous result [8] for a composition with slightly smaller carrier density. The $\mathrm{Cu}_{1.82} \mathrm{Te}{ }_{395}$ result is somewhat broader a more negative shift, while the $\mathrm{Cu}_{1.78} \mathrm{Te}$ line shape is very different corresponding to its modified crystal structure, and in line with the $\mathrm{Cu}$ NMR results described above.

${ }^{125}$ Te has spin $1 / 2$ and does not experience elec- 400 tric quadrupole broadening, in contrast to the $\mathrm{Cu}$ nuclei, and as a result the ${ }^{125} T_{1}$ is magnetic-only, dominated in this case by charge carriers. As shown in Fig. 5 for $\mathrm{Cu}_{1.82} \mathrm{Te}$ and $\mathrm{Cu}_{1.78} \mathrm{Te}, T_{1}$ decreases with increasing shift when sampled across the line 405 shapes. This indicates that a distribution of Knight shifts is a main feature determining the line shapes; similar results were obtained for $\mathrm{Cu}_{1.87} \mathrm{Te}$.

Regarding the Te Knight shifts, assuming $K=$ 0 at $\delta_{c s}=-1170 \mathrm{ppm}$, the mean value identified previously [8], with $T_{1}$ measured at line-center positions we obtain $K^{2} T_{1} T=2.7,2.5$, and $3.2 \times 10^{-6}$ sK for $\mathrm{Cu}_{1.78} \mathrm{Te}, \mathrm{Cu}_{1.82} \mathrm{Te}$, and $\mathrm{Cu}_{1.87} \mathrm{Te}$, respectively, compared to $2.6 \times 10^{-6} \mathrm{sK}$ for simple metallic behavior [36, 41]. The results help to confirm the assumption of Korringa behavior with small or 415 zero enhancement. Note however that the decrease in $T_{1}$ sampled across the split $\mathrm{Cu}_{1.78}$ Te line is not as large as expected for purely Korringa behavior. For example assuming $K^{2} T_{1} T=2.6 \times 10^{-6} \mathrm{sK}$ across

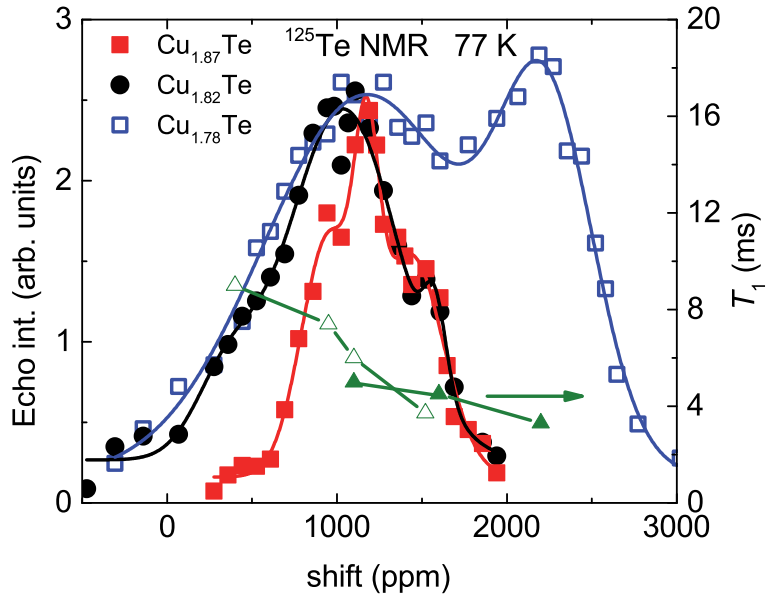

Figure 5: ${ }^{125}$ Te spectra for 3 samples measured at $77 \mathrm{~K}$. Solid curves from a fitted sum of 2 or 3 Gaussian curves intended to guide the eye. Also shown: $T_{1}$ measured at selected positions for $\mathrm{Cu}_{1.82} \mathrm{Te}$ (open triangles) and $\mathrm{Cu}_{1.78} \mathrm{Te}$ (solid triangles), plotted against right axis.

the line, $T_{1}$ for the left and right peaks (Fig. 5) gives $K=2600$ and $3200 \mathrm{ppm}$ respectively, and correspondingly $\delta_{c s}=-1500$ and -1000 , thus perhaps a $500 \mathrm{ppm}$ chemical shift contribution to the observed splitting. Similarly for $\mathrm{Cu}_{1.87} \mathrm{Te}$ assuming an unenhanced $K^{2} T_{1} T=2.6 \times 10^{-6} \mathrm{sK}$ gives a renormalized $\delta_{c s}=-1000 \mathrm{ppm}$, a small difference perhaps mainly responsible for its observed difference in peak position relative to $\mathrm{Cu}_{1.82} \mathrm{Te}$. Since $K$ contributions are large, these differences fall within the uncertainty of the analysis [8], however the large negative $\delta_{c s}$, along with Korringa behavior indicating a significant $s$-symmetry contribution to the valence band edge, are consistent with and reinforce the previous results giving evidence for topologically nontrivial behavior [8]. These shifts can be compared to observed ${ }^{125} \mathrm{Te}$ shifts for molecular systems falling in the range of about $\delta=-800$ to $+2300 \mathrm{ppm}[43,44]$, while recent reports in semiconductors for which $K$ contributions were believed small include ZnTe [45], -875 ppm, and the inverted-band [46] PbTe (-1150 ppm) and SnTe (-970 ppm), along with GeTe (-230 ppm), with all values renormalized according to shift reagent.

\subsection{Computational Results}

DFT calculations in the four structures discussed above $(\beta$-I, $\beta$-II, $\beta$-III, and $P \overline{3} m 1$ trigonal) were used to calculate electric field gradients (EFGs), and thus the $\mathrm{Cu}$ quadrupole NMR lineshapes. These methods have been shown to work 
well in computing quadrupole NMR parameters, often within 10-20\% of experimental values and thus providing a means to examine local structures [32]. Fig. 2(b-d) shows some of the results. Part 475 (b) of the figure shows the model curve obtained from the $\beta$-III structure calculation plotted with the $\mathrm{Cu}_{1.87} \mathrm{Te}{ }^{63} \mathrm{Cu}$ NMR data. The low-intensity curves in the figure are individual lines for each of the 24 $\mathrm{Cu}$ sites, while the upper curve is the summed line shape. A single overall scaling parameter was applied to match the amplitude to the experimental data. As noted above, the DFT calculations were performed for full site occupancy rather than with random vacancies included, and the major effect of localized vacancies on the NMR spectra will be a broadening process. The absence of the sharp peaks in the experimental spectra, as compared to the computed curves, is thus likely due to the distribution of vacancies in the samples. The match for the data in Fig. 2(b) is thus reasonable, including shifts which arise from sites having relatively large EFG tensors with nonzero asymmetry.

The calculation for the $P \overline{3} m 1$ trigonal structure is shown in Fig. 2(c), plotted with the $\mathrm{Cu}_{1.82} \mathrm{Te}$ results. This structure has only 4 inequivalent $\mathrm{Cu}$ sites. The generally smaller EFG's resulting from this structure lead to a smaller line width, and it appears that this structure does not fit the NMR results for either $\mathrm{Cu}_{1.87} \mathrm{Te}$ or $\mathrm{Cu}_{1.82} \mathrm{Te}$. Also, while 450 this method of computing EFG's is not relevant for the Te NMR results, the $P \overline{3} m 1$ trigonal structure with only two Te sites also does not agree with the Te NMR results, since the variation of $T_{1}$ across the line and the structure of the NMR spectra are in455 dicative of a superposition of three or more distinct sites. Thus the $\beta$-III structure provides the better match for these two compositions.

The two structures $\beta$-I and $\beta$-II provided the best agreement of XRD results to the $\mathrm{Cu}_{1.78} \mathrm{Te}$ 460 composition. Among these, in the computed $\beta$ II results all $\mathrm{Cu}$ sites have axial symmetry, hence an EFG tensor with relatively constrained secondorder quadrupole line shapes. The resulting $\mathrm{Cu} 510$ NMR spectrum has no intensity outside the range

$465-750$ to $+250 \mathrm{ppm}$, and thus provides a poor fit to the data. Note that anisotropic chemical and Knight shift contributions are not included in the calculated results, but these are expected to be considerably smaller that the observed width. On the other hand the calculation based on the $\beta$-I structure provided a reasonable match, as shown in Fig. 2(d). In modeling the results, different sites provide the main source of intensity for the two main observed peaks in the spectrum, a result which is reasonable given the different observed $T_{1}$ results for these positions.

\section{Conclusion}

We performed ${ }^{63} \mathrm{Cu},{ }^{65} \mathrm{Cu}$, and ${ }^{125} \mathrm{Te}$ NMR on $\mathrm{Cu}_{2-x}$ Te with $x$ between 0.13 and 0.22 , along with transport and structural measurements. The results agree with the phase diagram proposed by Baranova, with a phase change near $x=0.20$ fitted to a transition from the $\beta$-III structure for $x=$ $0.13-0.18$ to $\beta$-I for larger $x$. These compositions have been variously described as metallic or semiconducting, however the Hall and Korringa NMR behavior are shown here to correspond to filling of simple uncompensated hole pockets, behavior which extends across the phase boundary. The Korringa analysis also allows identification of the $\mathrm{Cu}$ and Te NMR chemical shifts, shown to fall at the far negative end of the expected shift ranges for all compositions. This behavior was previously identified with topologically nontrivial inverted band configuration. For the $x=0.22$ structure there is a large splitting in chemical shift corresponding to two rather different local environments for both $\mathrm{Cu}$ and Te sites. Furthermore the activation energy for initiation of $\mathrm{Cu}$ ion hopping near room temperao ture is rapidly reduced with increased $\mathrm{Cu}$ vacancies to $0.12 \mathrm{eV}$ for $x=0.22$. These barriers are considerably smaller than detected at higher temperatures, indicating a tail of relatively mobile ions which may have important consequences for device applications.

\section{Acknowledgement}

This work was supported by the Robert A. Welch Foundation, Grant No. A-1526, and also by the Department of Energy, with support under the Office of Basic Energy Sciences Award \# DE-SC-0008574.

\section{References}

[1] K. Sridhar, K. Chattopadhyay, Synthesis by mechanical alloying and thermoelectric properties of $\mathrm{Cu}_{2} \mathrm{Te}, \mathrm{J}$. Alloys Compd. 264 (1998) $293-298$.

[2] H. Liu, X. Shi, F. Xu, L. Zhang, W. Zhang, L. Chen, Q. Li, C. Uher, T. Day, G. J. Snyder, Copper ion liquidlike thermoelectrics, Nature Mater. 11 (2012) 422. 
[3] S. Ballikaya, H. Chi, J. R. Salvador, C. Uher, Thermoelectric properties of Ag-doped $\mathrm{Cu}_{2} \mathrm{Se}$ and $\mathrm{Cu}_{2} \mathrm{Te}, \mathrm{J}$. Mater. Chem. A 1 (2013) 12478.

[4] Y. He, T. Zhang, X. Shi, S.-H. Wei, L. Chen, High thermoelectric performance in copper telluride, NPG Asia Mater. 7 (2015) e210.

[5] J. H. Yun, K. H. Kim, D. Y. Lee, B. T. Ahn, Back contact formation using $\mathrm{Cu}_{2} \mathrm{Te}$ as a $\mathrm{Cu}$-doping source 590 and as an electrode in CdTe solar cells, Solar Energy Mater. Solar Cells 75 (2003) $203-210$.

[6] M. C. Nguyen, J.-H. Choi, X. Zhao, C.-Z. Wang, Z. Zhang, K.-M. Ho, New layered structures of cuprous chalcogenides as thin film solar cell materials: $\mathrm{Cu}_{2} \mathrm{Te}{ }_{595}$ and $\mathrm{Cu}_{2}$ Se, Phys. Rev. Lett. 111 (2013) 165502.

[7] A. C. Poulose, S. Veeranarayanan, M. S. Mohamed, R. R. Aburto, T. Mitcham, R. R. Bouchard, P. M. Ajayan, Y. Sakamoto, T. Maekawa, D. S. Kumar, Multifunctional $\mathrm{Cu}_{2-\mathrm{x}}$ Te nanocubes mediated combination 600 therapy for multi-drug resistant MDA MB 453, Sci. Rep. 6 (2016) 35961.

[8] A. A. Sirusi, S. Ballikaya, J.-H. Chen, C. Uher, J. H. Ross, Band ordering and dynamics of $\mathrm{Cu}_{2-\mathrm{x}} \mathrm{Te}$ and $\mathrm{Cu}_{1.98} \mathrm{Ag}_{0.2} \mathrm{Te}$, J. Phys. Chem. C 120 (27) (2016) 605 14549-14555.

[9] Y. Ma, L. Kou, Y. Dai, T. Heine, Two-dimensional topological insulators in group-11 chalcogenide compounds: $M_{2} \mathrm{Te}(M=\mathrm{Cu}, \mathrm{Ag})$, Phys. Rev. B 93 (2016) 235451 .

[10] W. Zhang, R. Yu, W. Feng, Y. Yao, H. Weng, X. Dai, $\mathrm{Z}$. Fang, Topological aspect and quantum magnetoresistance of $\beta-\mathrm{Ag}_{2}$ Te, Phys. Rev. Lett. 106 (2011) 156808

[11] S. Lee, J. In, Y. Yoo, Y. Jo, Y. C. Park, H. jun Kim H. C. Koo, J. Kim, B. Kim, K. L. Wang, Single crys- 6 talline $\beta-\mathrm{Ag}_{2}$ Te nanowire as a new topological insulator, Nano Lett. 12 (2012) 4194-4199.

[12] A. Sulaev, W. Zhu, K. L. Teo, L. Wang, Gate-tuned quantum oscillations of topological surface states in $\beta-$ $\mathrm{Ag}_{2}$ Te, Sci. Rep. 5 (2015) 8062.

[13] J. Kim, A. Hwang, S.-H. Lee, S.-H. Jhi, S. Lee, Y. C. Park, S.-i. Kim, H.-S. Kim, Y.-J. Doh, J. Kim, B. Kim, Quantum electronic transport of topological surface states in $\beta-\mathrm{Ag}_{2}$ Se nanowire, ACS Nano 10 (2016) 3936-3943.

[14] Y. G. Asadov, L. V. Rustamova, G. B. Gasimov, K. M. Jafarov, A. G. Babajev, Structural phase transitions in $\mathrm{Cu}_{2-\mathrm{x}}$ Te crystals $(x=0.00,0.10,0.15,0.20,0.25)$, Phase Transit. 38 (1992) 247-259.

[15] R. Blachnik, M. Lasocka, U. Walbrecht, The system 630 copper-tellurium, J. Solid State Chem. 48 (1983) 431 438.

[16] J. Boyce, B. Huberman, Superionic conductors: Transitions, structures, dynamics, Phys. Rep. 51 (1979) 189265.

[17] H. Nowotny, Die kristallstructur von $\mathrm{Cu}_{2}$ Te, Z. Metallkd. 37 (1946) 40.

[18] I. Patzak, Uber die struktur und die lage der phasen im system kupfer-tellur, Z. Metallkd. 47 (1956) 418

[19] S. Matar, R. Weihrich, D. Kurowski, A. Pfitzner, DFT 640 calculations on the electronic structure of $\mathrm{CuTe}_{2}$ and $\mathrm{Cu}_{7} \mathrm{Te}_{4}$, Solid State Sci. 6 (2004) 15-20.

[20] D. P. D. Kurowski, Ph.D. thesis, University of Regensburg, Germany (2003).

[21] L. Yu, K. Luo, S. Chen, C.-G. Duan, Cu-deficiency 645 induced structural transition of $\mathrm{Cu}_{2-x} \mathrm{Te}$, CrystEngComm 17 (14) (2015) 2878-2885.
[22] R. V. Baranova, A. S. Avilov, Z. G. Pinsker, Determination of crystal-structure of hexagonal phase $\beta$-III in $\mathrm{Cu}$-Te system by electron-diffraction method, Kristallografiya 18 (1973) 1169.

[23] R. V. Baranova, Electron diffraction determination of the structure of the $\beta$-I phase in the $\mathrm{Cu}$-Te system, Kristallografiya 12 (1967) 266

[24] R. V. Baranova, Electron diffraction determination of the crystal structure of the hexagonal phase $\beta$-II in the Cu-Te system, Kristallografiya 13 (1968) 803.

[25] R. V. Baranova, Crystal structure determination by electron diffraction of the hexagonal $\beta$ double phase in the $\mathrm{Cu}-\mathrm{Te}$ system, Sov. Phys. Crystallogr. 13 (1969) 695-699.

[26] B. H. Toby, EXPGUI, a graphical user interface for GSAS, J. Appl. Crystallogr. 34 (2001) 210-213.

[27] A. C. Larson, R. B. V. Dreele, Los Alamos National Laboratory Report LAUR (1994) 86-748.

[28] M. Inamo, ${ }^{125} \mathrm{Te} \mathrm{NMR}$ evidence for the existence of trinuclear tellurate ion in aqueous solution, Chem. Lett. 25 (1996) 17-18.

[29] D. Massiot, F. Fayon, M. Capron, I. King, S. Le Calvé, B. Alonso, J.-O. Durand, B. Bujoli, Z. Gan, G. Hoatson, Modelling one- and two-dimensional solid-state NMR spectra, Magn. Reson. Chem. 40 (2002) 70-76.

[30] A. A. Sirusi, J. H. Ross, Pseudogap and anharmonic phonon behavior in $\mathrm{Ba}_{8} \mathrm{Ga}_{16} \mathrm{Ge}_{30}$ : An NMR study, J. Chem. Phys. 145 (2016) 054702.

[31] P. Blaha, K. Schwarz, G. Madsen, D. Kvasnicka, J. Luitz, WIEN2k, an Augmented Plane Wave+Local Orbitals Program for Calculating Crystal Properties, Technische Universität Wien, Austria, 2001.

[32] K. Schwarz, P. Blaha, Solid state calculations using WIEN2k, Comput. Mater. Sci. 28 (2003) 259-273.

[33] J. P. Perdew, K. Burke, M. Ernzerhof, Generalized gradient approximation made simple, Phys. Rev. Lett. 77 (1996) 3865-3868

[34] S. Kashida, W. Shimosaka, M. Mori, D. Yoshimura, Valence band photoemission study of the copper chalcogenide compounds, $\mathrm{Cu}_{2} \mathrm{~S}, \mathrm{Cu}_{2} \mathrm{Se}$ and $\mathrm{Cu}_{2} \mathrm{Te}$, J. Phys. Chem. Solids 64 (2003) 2357-2363.

[35] Y. Zhang, Y. Wang, L. Xi, R. Qiu, X. Shi, P. Zhang, W. Zhang, Electronic structure of antifluorite $\mathrm{Cu}_{2} \mathrm{X}(X$ $=\mathrm{S}$, Se, Te) within the modified Becke-Johnson potential plus an on-site Coulomb $U$, J. Chem. Phys. 140 (2014) 074702.

[36] J. Korringa, Nuclear magnetic relaxation and resonnance line shift in metals, Physica 16 (1950) $601-610$.

[37] X. Zheng, S. Y. Rodriguez, J. H. Ross, Jr., NMR relaxation and rattling phonons in the type-I $\mathrm{Ba}_{8} \mathrm{Ga}_{16} \mathrm{Sn}_{30}$ clathrate, Phys. Rev. B 84 (2011) 024303.

[38] K. Wakamura, Origin of the low-energy mode in superionic conductors, Phys. Rev. B 59 (1999) 3560-3568.

[39] S.-Y. Miyatani, S. Mori, M. Yanagihara, Phase diagram and electrical properties of $\mathrm{Cu}_{2-\delta}$ Te, J. Phys. Soc. Jpn. 47 (1979) 1152-1158.

[40] R. A. Yakshibaev, N. N. Mukhamadeeva, R. F. Almukhametov, Phase transformations and ionic transport in the $\mathrm{Cu}_{2-\delta}$ Te superionic conductor, Phys. Status Solidi (a) 108 (1988) 135-141.

[41] G. C. Carter, L. H. Bennett, D. J. Kahan, Metallic shifts in NMR, Prog. Mater. Sci. 20

[42] Y. Zhang, B. Sa, J. Zhou, Z. Sun, First principles investigation of the structure and electronic properties of $\mathrm{Cu}_{2} \mathrm{Te}$, Comp. Mater. Sci. 81 (2014) 163-169. 
[43] Y. Ruiz-Morales, G. Schreckenbach, T. Ziegler, Calculation of ${ }^{125} \mathrm{Te}$ chemical shifts using gauge-including atomic orbitals and density functional theory, J. Phys. Chem. A 101 (22) (1997) 4121-4127.

[44] H. Günther, NMR Spectroscopy: Basic Principles, Concepts and Applications in Chemistry, Wiley-VCH, Siegen, Germany, 2013.

[45] D. Koumoulis, G. D. Morris, L. He, X. Kou, D. King, D. Wang, M. D. Hossain, K. L. Wang, G. A. Fiete, M. G. Kanatzidis, L.-S. Bouchard, Nanoscale $\beta$-nuclear magnetic resonance depth imaging of topological insulators, Proc. Natl. Acad. Sci. USA 112 (28) (2015) E3645E3650.

660

[46] B. Njegic, E. Levin, K. Schmidt-Rohr, ${ }^{125}$ Te NMR chemical-shift trends in PbTe-GeTe and PbTe-SnTe alloys, Solid State Nucl. Mag. Reson. 55-56 (2013) 79-83. 